TITLE:

\title{
Helical rearrangement of photoactivated rhodopsin in monomeric and dimeric forms probed by high-angle X-ray scattering
}

\section{AUTHOR(S):}

Imamoto, Yasushi; Kojima, Keiichi; Oka, Toshihiko; Maeda, Ryo; Shichida, Yoshinori

\section{CITATION:}

Imamoto, Yasushi ... [et al]. Helical rearrangement of photoactivated rhodopsin in monomeric and dimeric forms probed by high-angle X-ray scattering. Photochemical and Photobiological Sciences 2015, 14: 1965-1973

ISSUE DATE:

2015-08-05

URL:

http://hdl.handle.net/2433/209998

\section{RIGHT:}

This is the accepted version of the article, which has been published in final form at

http://dx.doi.org/10.1039/C5PP00175G.; The full-text file will be made open to the public on 5 August 2016 in

accordance with publisher's 'Terms and Conditions for Self-Archiving'; この論文は出版社版でありません。引用の際に

は出版社版をご確認ざ利用ください。; This is not the published version. Please cite only the published version. 


\title{
Helical Rearrangement of Photoactivated Rhodopsin in Monomeric and Dimeric Forms Probed by High-Angle X-Ray Scattering ${ }^{\dagger}$
}

Yasushi Imamoto, ${ }^{* a}$ Keiichi Kojima, ${ }^{a}$ Toshihiko Oka, ${ }^{\text {bc }}$ Ryo Maeda, ${ }^{\mathrm{a}}$ and Yoshinori Shichida ${ }^{\mathrm{a}}$

aDepartment of Biophysics, Graduate School of Science, Kyoto University, Kyoto 606-8502, Japan.

E-mail: imamoto@rh.biophys.kyoto-u.ac.jp

${ }^{b}$ Department of Physics, Faculty of Science, Shizuoka University, Shizuoka 422-8529, Japan.

'Nanomaterials Research Division, Research Institute of Electronics, Shizuoka University, Shizuoka 432-8011, Japan.

†This manuscript is submitted as part of a themed issue on the 16th International Conference on Retinal Proteins.

\begin{abstract}
Light-induced helical rearrangement of vertebrate visual rhodopsin was directly monitored by high-angle X-ray scattering (HAXS), ranging from $Q(=4 \pi \sin \theta / \lambda)=0.03 \AA^{-1}$ to $Q=1.5 \AA^{-1}$. HAXS of nanodiscs containing single a rhodopsin molecule was performed before and after photoactivation of rhodopsin. The intensity difference curve obtained by HAXS agreed with that calculated from the crystal structure of dark state rhodopsin and metarhodopsin II, indicating that the conformational change of monomeric rhodopsin in the membrane is consistent with that occurring in the crystal. On the other hand, the HAXS intensity difference curve of nanodiscs containing two rhodopsin molecules was significantly reduced, similar to that calculated from the crystal structure of the deprotonated intermediate, without a large conformational change. These results suggest that rhodopsin is dimerized in the membrane, and that the interaction between rhodopsin molecules affects structural changes.
\end{abstract}




\section{Introduction}

Rhodopsin, which is located in the outer segment of rod visual cells in animal retinas, is a G-protein-coupled receptor (GPCR). Rhodopsin activates the G-protein transducin $\left(\mathrm{G}_{t}\right)$ in response to a photon. Structurally GPCRs share a common structural motif composed of seven transmembrane helices. ${ }^{1}$ Upon activation by stimuli, GPCRs undergo helical rearrangement, resulting in a conformational change, enabling them to interact with their cognate $\mathrm{G}$ proteins on the cytoplasmic surface..$^{2-10}$

Rhodopsin is composed of the apoprotein opsin and the light absorbing chromophore 11cis-retinal, which covalently binds to Lys residue of opsin via a Schiff base linkage. The Schiff base linkage is protonated to delocalize the $\pi$-electron system of the chromophore, which enables rhodopsin to absorb visible light. In dark state rhodopsin, the 11-cis-retinal chromophore acts as an inverse-agonist, which stabilizes the inactive conformation, substantially reducing G-protein activation efficiency. ${ }^{11}$ On photon absorption, the 11-cisretinal chromophore is isomerized to the all-trans form. After photoisomerization of the chromophore, a series of photobleaching intermediates such as bathorhodopsin, lumirhodopsin, metarhodopsin I, metarhodopsin II and metarhodopsin III is formed. In metarhodopsin II (Meta-II), a proton is released from the Schiff base linkage. As the protonated Schiff base is a core of the intramolecular hydrogen-bonding network, deprotonation of the Schiff base results in a substantial conformational change, resulting in the formation of the active state.

To date these helical rearrangements of GPCRs have been studied by various kinds of spectroscopy, ${ }^{12-16}$ as well as X-ray/electron crystallography. ${ }^{3-6,9,}{ }^{10}$ However, spectroscopy mainly provides highly localized information around the functional group. While crystallography shows the overall structure in detail, the protein structure in the crystal might be different from the native structure due to crystal packing. Recently, it was reported that solution X-ray scattering in the high-angle region $\left(Q<1.5 \AA^{-1}\right.$, where momentum transfer $Q$ $=4 \pi \sin \theta / \lambda)$ is a powerful tool to analyze helical rearrangements in seven transmembrane proteins, including rhodopsin ${ }^{17}$ and bacteriorhodopsin. ${ }^{18}$

Solution X-ray scattering experiments have been mainly applied to the small-angle region, in which the Guinier approximation $\left(\ln I(Q)=\ln I(0)-\frac{R_{\mathrm{g}}{ }^{2}}{3} Q^{2}\right.$, where $I(Q)$ is scattering intensity at $Q, I(0)$ represents forward scattering, and $R_{\mathrm{g}}$ is the radius of gyration), 
is valid. Using this approximation, $R \mathrm{~g}$, which is the parameter showing the dimension of the scatterer, is obtained. Therefore, Guinier analysis is used to observe large-scale conformational changes, such as protein unfolding. ${ }^{19}$ On the other hand, information obtained from high-angle X-ray scattering (HAXS) corresponds to the distance between secondary structure elements. Therefore, fine conformational changes can be detected by HAXS, even if the conformational change is too small to cause any detectable change in $R_{\mathrm{g}}$.

In this study, we directly monitored light-induced helical rearrangements of rhodopsin by HAXS. To address the long-standing issue of the dimerization of GPCRs, the effect of dimerization on conformational changes was assessed. The light-induced conformational change of the rhodopsin monomer, isolated in the nanodisc, was compared to that of the dimer in the nanodisc, or detergent-solubilized rhodopsin. The results demonstrated that the dimerization affects the extent of conformational changes, suggesting that an interaction between the rhodopsin molecules tethered in the nanodisc may modulate conformational change.

\section{Experimental}

\subsection{Sample preparation}

Bovine rod outer segments were prepared from bovine fresh retina. Retinas were removed from eye balls under dim red light. They were suspended in $40 \%$ sucrose (w/v) in ROS buffer (10 mM MOPS, $30 \mathrm{mM} \mathrm{NaCl}, 60 \mathrm{mM} \mathrm{KCl,} 2 \mathrm{mM} \mathrm{MgCl}_{2}, 1 \mathrm{mM}$ dithiothreitol, $\mathrm{pH}$ 7.5), vigorously shaken manually and centrifuged. The floating material and supernatant was twice diluted by ROS buffer, and centrifuged to collect ROS as the pellet. This manipulation was repeated 2 to 3 times.

Crude ROS was suspended in a small volume of ROS buffer, and layered on a $29 \% / 35 \%$ (w/v in ROS buffer) sucrose stepwise gradient. After centrifugation, ROS in the interface between 29\% and 35\% sucrose was collected, and washed with ROS buffer several times.

The ROS membrane suspension was separated into three portions. Rhodopsin in one portion was solubilized by $1 \%$ dodecyl maltoside (DDM), and adsorbed on Concanavalin A Sepharose. After washing with DDM buffer (0.02\% DDM, $50 \mathrm{mM}$ HEPES, $140 \mathrm{mM} \mathrm{NaCl}$, $\mathrm{pH}$ 6.0), rhodopsin was eluted by adding $300 \mathrm{mM} \alpha$-methyl-D-mannopyranoside in DDM buffer, and concentrated to $30 \mathrm{mg} / \mathrm{mL}$ by ultrafiltration membrane (Centricon 30, Millipore). 
The other portions of ROS membrane suspension were used to prepare nanodisc (ND) samples. ${ }^{20,21}$ Rhodopsin was solubilized with OG buffer (1\% n-octyl- $\beta$-D-glucoside, $20 \mathrm{mM}$ Tris/HCl, $100 \mathrm{mM} \mathrm{NaCl}, 0.5 \mathrm{mM}$ EDTA, pH 7.4). Membrane scaffold protein (MSP) MSP1E3D1 was expressed in Escherichia coli, and purified by Ni-chelate affinity column chromatography. ${ }^{20}$ Rhodopsin, MSP1E3D1, and 1-palmitoyl-2-oleoyl-sn-glycero-3phosphocholine (POPC) were mixed at a molar ratio of 750:10:1 (lipids:MSP:pigment), to prepare ND containing a single rhodopsin molecule (Rh/ND), or 55:1:1 to prepare ND containing two rhodopsin molecules (2Rh/ND). ${ }^{22} \mathrm{ND}$ was self-assembled by removing OG with dialysis against Buffer $\mathrm{C}(20 \mathrm{mM}$ Tris/ $\mathrm{HCl}, 100 \mathrm{mM} \mathrm{NaCl}, 0.5 \mathrm{mM}$ EDTA, $\mathrm{pH}$ 7.4) at $4{ }^{\circ} \mathrm{C}$. The aggregates and liposomes were removed from the mixture using a Superdex 200 column chromatography. The collected fractions contained empty ND, as well as Rh/ND or 2Rh/ND. To remove empty ND, Concanavalin A Sepharose was added to the sample. After washing the resin several times, $\mathrm{Rh} / \mathrm{ND}$ or $2 \mathrm{Rh} / \mathrm{ND}$ was eluted by $300 \mathrm{mM} \alpha$-methyl-Dmannopyranoside in buffer D (50 mM HEPES, $140 \mathrm{mM} \mathrm{NaCl}, \mathrm{pH} 6.0)$. After the buffer was exchanged to buffer D by PD-10 column, the sample was concentrated to $7 \mathrm{mg} / \mathrm{mL}$ rhodopsin $(\mathrm{Rh} / \mathrm{ND})$ or $17 \mathrm{mg} / \mathrm{mL}$ rhodopsin $(2 \mathrm{Rh} / \mathrm{ND})$ by ultrafiltration membrane (Centricon 30, Millipore). The stoichiometry of rhodopsin $\left(\varepsilon_{500 \mathrm{~nm}}=40,600 \mathrm{M}^{-1} \mathrm{~cm}^{-1}\right)$ and MSP1E3D1 $\left(\varepsilon 280 \mathrm{~nm}=29,910 \mathrm{M}^{-1} \mathrm{~cm}^{-1}\right)$ was estimated to be $1: 2$ for $\mathrm{Rh} / \mathrm{ND}$, and 1:0.9 for $2 \mathrm{Rh} / \mathrm{ND}$, by UVvisible absorption spectra. Because one ND contains two MSP, ND in Rh/ND and 2Rh/ND preparations dominantly contains one or two rhodopsin molecules, respectively.

Empty ND for the control experiment was prepared by dialyzing the 1:75 mixture of MSP and POPC, followed by Superdex 200 column chromatography.

\subsection{Time-resolved UV-visible absorption spectroscopy}

The photoreaction of rhodopsin was measured using a multichannel CCD spectrometer (C10000 system, Hamamatsu Photonics). ${ }^{23}$ Rhodopsin in the sample was photoactivated by a yellow flash generated by a short-arc xenon flash lamp (SA200, Nissin Electronic) and a glass optical filter (Y52, Asahi Techno Glass). The transient spectra were recorded every 100 $\mu \mathrm{s}$, and analyzed by singular value decomposition method ${ }^{21,24}$ executed on Igor Pro 6.3. (WaveMetrix).

\subsection{High-angle $X$-ray scattering}

High-angle X-ray scattering (HAXS) was measured at the beam line BL40B2 in SPring-8 
(Hyogo, Japan). The sample $(25 \mu \mathrm{L})$ was placed into a 2-mm-diameter quartz capillary (Hilgenberg 4017250), and illuminated from the top of the sample cell holder to photoactivate rhodopsin. For photoactivation of rhodopsin, yellow light (>500 nm) was generated by a $150-\mathrm{W}$ cold light source (HL 150, Hoya-Schott), and passed through a glass optical filter (Y52, Asahi Techno Glass). The temperature of the sample was maintained at $20{ }^{\circ} \mathrm{C}$ by circulating the temperature-controlled water in the capillary holder.

The concentric scattering image was obtained by R-AXIS VII (Rigaku), whose detector surface was $540 \mathrm{~mm}$ from the sample. The of X-ray wavelength was $1.0 \AA$, and the beam size was $0.4(\mathrm{w}) \times 0.2(\mathrm{~h}) \mathrm{mm}$. The sample was exposed to X-ray for $60 \mathrm{~s}$ to obtain one scattering image, and it took about $120 \mathrm{~s}$ for readout. The scattering curve ( $I$ vs $Q$ ) was calculated from the circular average of the scattering image. To reduce radiation damage, the sample stage was reciprocally moved $( \pm 0.5 \mathrm{~mm}, 0.5 \mathrm{~Hz})$. Four scattering images were taken before photoactivation of rhodopsin. The sample was then irradiated for $30 \mathrm{~s}$ with yellow light, and four scattering images were taken. The amount of photoactivated rhodopsin was estimated by UV-visible absorption spectra, measured using a Shimadzu UV2400PC spectrophotometer.

\section{Results and Discussion}

\subsection{Photoreaction of rhodopsin in DDM and nanodiscs}

It has been reported that a metastable equilibrium mixture composed of Meta-I and MetaII is produced by the photoactivation of rhodopsin, and then Meta-III is slowly formed. ${ }^{25}$ It is well established that acidic $\mathrm{pH}$ and DDM biases this equilibrium to Meta-II. ${ }^{26,27}$ In ND, the fraction of Meta-II, as well as the $\mathrm{G}_{t}$ activation efficiency of monomeric rhodopsin (Rh/ND), is greater than those of dimeric ones $(2 \mathrm{Rh} / \mathrm{ND})^{28}$. Here, we compared the reactions of $\mathrm{Rh} / \mathrm{DDM}, \mathrm{Rh} / \mathrm{ND}$ and $2 \mathrm{Rh} / \mathrm{ND}$ at $\mathrm{pH}$ 6.0, in detail, by transient UV-visible absorption spectroscopy (Figure 1).

$\mathrm{Rh} / \mathrm{DDM}, \mathrm{Rh} / \mathrm{ND}$, and $2 \mathrm{Rh} / \mathrm{ND}$ were excited by yellow flash, and transient difference spectra were recorded every $100 \mu \mathrm{s}$. Next the decay-associated difference spectra (DADS) were calculated by singular value decomposition analysis. DADS are exponential decay components, which essentially show the difference spectra between the photobleaching intermediates. The bleaching processes of $\mathrm{Rh} / \mathrm{DDM}, \mathrm{Rh} / \mathrm{ND}$, and $2 \mathrm{Rh} / \mathrm{ND}$, over a $10^{-4}$ to $10^{3}$ sec time scale, were represented by three processes (DADS1-DADS3) (Figure 1). In all samples, DADS1 and DADS2 showed a negative peak at $480 \mathrm{~nm}$ and positive peak at 380 
$\mathrm{nm}$, indicating that Meta-I heterologously decays to Meta-II. The time constants $(\tau=1 / k)$ for DADS1 and DADS2 of Rh/DDM were about $1 / 10$ those of $\mathrm{Rh} / \mathrm{ND}$, whereas those of 2Rh/ND were 2 to 3 times greater than those of Rh/ND (Table 1). DADS3 showed a negative peak at $380 \mathrm{~nm}$ and positive peak at $450 \mathrm{~nm}$, indicating the slow formation of Meta-III. The time constants for formation of Meta-III were not largely different from each other (Table 1). Because we did not observe the decay of Meta-III, the extrapolated spectra at infinite time (DADS0) show the difference spectra between dark state and the equilibrium mixture composed of Meta-I, Meta-II, and Meta-III.

Environmental effects on the equilibrium between Meta-I and Meta-II were assessed by analyzing the transient spectrum. Meta-I decays to Meta-II in the double-exponential reaction to form the equilibration mixture (DADS1 and DADS2), and then Meta-III is exponentially formed from the Meta-I/Meta-II mixture (DADS3). Therefore, the spectrum of Meta-I/MetaII equilibrium is obtained by subtracting DADS3 from DADS0.

Obtained spectra (Figure 2, red) were decomposed into the model difference spectra of Meta-I minus rhodopsin (green), and Meta-II minus rhodopsin (blue) to estimate the ratio of Meta-I and Meta-II (Figure 2 and Table 1). The resulting spectra (black) were in good agreement with the experimental curves (red). As reported previously, DDM stabilizes MetaII, whereas Meta-I is stabilized in 2Rh/ND. The slow formation of Meta-II, as well as the shift of the equilibrium in $2 \mathrm{Rh} / \mathrm{ND}$ in comparison with $\mathrm{Rh} / \mathrm{ND}$, suggests an interaction between the rhodopsin molecules tethered in the ND.

\subsection{X-ray scattering profile of rhodopsin in DDM and nanodiscs}

To obtain HAXS curves, scattering images were taken by R-AXIS VII, with 1-min exposure, and the concentric images were circularly averaged. The overall scattering curves of $\mathrm{Rh} / \mathrm{DDM}, \mathrm{Rh} / \mathrm{ND}, 2 \mathrm{Rh} / \mathrm{ND}$, and empty ND are shown in Figure 3. The intensity of HAXS region was significantly smaller than that of the small-angle region $\left(\mathrm{Q}<0.1 \AA^{-1}\right)$.

ND is composed of membrane scaffold protein (MSP) and lipid bilayer. Since MSP is an $\alpha$-helical protein, the scattering from MSP may overlap with that of the $\alpha$-helix of rhodopsin. In the scattering curves of $\mathrm{Rh} / \mathrm{DDM}, \mathrm{Rh} / \mathrm{ND}$, and $2 \mathrm{Rh} / \mathrm{ND}$, a characteristic shoulder was observed at $Q=0.65 \AA^{-1}$ but not in empty ND, suggesting that the shoulder at $Q=0.65 \AA^{-1}$ represents the interhelical interference of rhodopsin molecule. ${ }^{29}$ To confirm this, the scattering curve was predicted from the crystal structure of rhodopsin. The scattering curve of monomeric rhodopsin in the dark state was calculated using the coordinates of polypeptide 
(Met1-Ala348) and 11-cis-retinal, in a-chain of $1 \mathrm{U} 19$ by $\mathrm{CRYSOL}^{30}$ (Figure 3). The scattering curve of antiparallel dimer was similarly calculated using a- and b-chains and 11cis-retinal of $1 \mathrm{U} 19$. The shoulder at $Q=0.65 \AA^{-1}$ was also present in these calculated curves, indicating that the packing of helices can be analyzed by HAXS. The shoulder is more prominent in $2 \mathrm{Rh} / \mathrm{ND}$ than in $\mathrm{Rh} / \mathrm{ND}$, possibly due to the high ratio of rhodopsin to MSP $(2: 2$ for $\mathrm{Rh} / \mathrm{ND}$ and 1:2 for $\mathrm{Rh} / \mathrm{ND}$ ). As $2 \mathrm{Rh} / \mathrm{ND}$ contains parallel and antiparallel dimers in the ratio of $1: 1,{ }^{31}$ the scattering curve of $2 \mathrm{Rh} / \mathrm{ND}$ represents the sum of the both dimers.

\subsection{Radiation Damage}

HAXS curves were measured before and after photoactivation of rhodopsin, but the difference was found to be very small (Figure 3). To improve the signal-to-noise ratio in the limited amount of sample, measurements were repeated four times, before and after photoactivation. The the intensity changes upon photoactivation were then calculated using the averaged curves. These measurements were independently carried out 3 times for Rh/ND and empty ND (Figures $4 a$ and $4 b)$.

For $\mathrm{Rh} / \mathrm{ND}$, the intensity difference curves in the $Q$ region from 0.4 to $1.2 \AA^{-1}$ were reproducible. However, those below $0.4 \AA^{-1}$ were not reproducible for $\mathrm{Rh} / \mathrm{ND}$, and a significant scattering change was observed for empty ND. These findings indicate that the scattering changes caused by the degradation of protein, and/or the ND by X-ray radiation are not negligible. Calculation of the different intensity curves between one measurement before illumination, and one measurement after illumination improved reproducibility, but the scattering changes caused by the X-ray radiation damage were still observed. Thus, we attempted to separate the X-ray scattering changes derived from structural change from those due to radiation damage (Figure $4 \mathrm{c}$ ).

The scattering intensities at given $Q$ in four scattering curves before photoactivation were plotted against the number of measurements $(n=1,2,3,4)$. They were fitted with a linear line, and the intensity just before the photoactivation $(n=4.5)$ was extrapolated. Similarly, the intensities at $Q$ in four scattering curves after photoactivation were plotted against the number of measurements $(n=5,6,7,8)$, fitted with a linear line, and the intensity just after the photoactivation $(n=4.5)$ was extrapolated. Using these extrapolated intensities, the scattering intensity changes $(\Delta l)$ at $Q$ were calculated, and plotted against $Q$ (Figures $4 \mathrm{~d}$ and $4 \mathrm{e})$.

While the signal-to-noise worsened, the reproducibility was found to be substantially 
improved for $\mathrm{Rh} / \mathrm{ND}$. In addition, the flat baseline obtained from the empty ND implies that radiation damage can be removed by means of such data processing. The HAXS curves shown below were obtained by these calculations.

\subsection{High-angle X-ray scattering}

The intensity difference curves upon photoactivation of rhodopsin in $\mathrm{Rh} / \mathrm{DDM}, \mathrm{Rh} / \mathrm{ND}$ and $2 \mathrm{Rh} / \mathrm{ND}$ were obtained, as described in the previous section (Figure 5). Under these experimental conditions ( $\mathrm{pH}$ 6.0, $20^{\circ} \mathrm{C}$ ), Meta-II was dominantly formed, but within the equilibrium mixture containing Meta-I and Meta-III. ${ }^{25}$ Time-resolved UV-visible absorption spectroscopy demonstrated that the percentages of Meta-I in Meta-I/Meta-II equilibrium were $13 \%$ in $\mathrm{Rh} / \mathrm{DDM}, 20 \%$ in $\mathrm{Rh} / \mathrm{ND}$, and $30 \%$ in $2 \mathrm{Rh} / \mathrm{ND}$ (Table 1). In addition, $\tau_{3}$ of these samples (Table 1) indicated that $40-50 \%$ of photoactivated rhodopsin was converted to MetaIII during the X-ray scattering measurements (10 min). However, because the intensity difference curves just after photoactivation were extrapolated (see Figure 4c), the generation of Meta-III during the X-ray scattering measurements was not taken into consideration. We assumed that the scattering change for the formation of Meta-I was significantly smaller than that of Meta-II, based on the low-resolution structure of Meta- $\mathrm{I}^{32}$ and recent time-resolved HAXS measurements ${ }^{17}$. It should be noted that Meta-II consists of multiple substates with different conformations (Meta- $\mathrm{II}_{\mathrm{a}}$ and Meta- $\mathrm{II}_{\mathrm{b}}$ ), and it is likely that the Meta- $\mathrm{II}_{\mathrm{b}}$ is dominantly formed under the present experimental conditions $\left(\mathrm{pH} 6,20^{\circ} \mathrm{C}\right) .{ }^{14,33}$ The intensity of HAXS was normalized by the amount of Meta-II formed by photoactivation, which was estimated from the percentages of photoactivated rhodopsin (70-87\%), and the equilibrium constant between Meta-I and Meta-II (Table 1).

In the intensity difference curves of $\mathrm{Rh} / \mathrm{DDM}, \mathrm{Rh} / \mathrm{ND}$, and $2 \mathrm{Rh} / \mathrm{ND}$, great intensity changes were observed in the small-angle region $\left(Q<0.2 \AA^{-1}\right)$ (Figure 5). The intensity change in this region is likely to reflect the overall shape of ND or DDM micelle. In addition, it was pointed out that phospholipids affect the scattering profile in this region. ${ }^{17,} 18$ Therefore, we examined scattering changes in $Q>0.2 \AA^{-1}(D<\sim 30 \AA)$, to focus on the helical rearrangements of rhodopsin.

The intensity difference curve of $\mathrm{Rh} / \mathrm{DDM}$ showed a sharp peak at $Q=0.2 \AA^{-1}$, and broad valley at $0.6 \AA^{-1}$, the latter of which corresponds to the characteristic shoulder showing the packing of helices (Figure 3). The intensity difference curve of Rh/ND essentially agreed 
with that of $\mathrm{Rh} / \mathrm{DDM}$, while the peak at $0.2 \AA^{-1}$ was greater than that in $\mathrm{Rh} / \mathrm{DDM}$. The intensity difference curve of $2 \mathrm{Rh} / \mathrm{ND}$ was similar to that of $\mathrm{Rh} / \mathrm{DDM}$, rather than that of $\mathrm{Rh} / \mathrm{ND}$. Because these scattering changes were not observed in empty $\mathrm{ND}$, it was concluded that they are derived from the conformational change of rhodopsin.

To date, conformational changes of rhodopsin during the photobleaching process have been studied in detail by X-ray/electron crystallography. While the conformational changes upon the formation of bathorhodopsin, lumirhodopsin, and Meta-I are small, ${ }^{32,} 34,35$ significant helical rearrangement is found to occur in Meta-II. ${ }^{5-8}$ When rhodopsin is photoactivated in solution and then crystallized, fully activated structures are obtained.$^{5-7}$ On the other hand, if deprotonated intermediate is produced by irradiation of the crystal in dark state, the helical rearrangements are limited due to crystal packing. ${ }^{8}$ This structure (2I37) represents the model structure of Meta- $\mathrm{II}_{\mathrm{a}}$, in which the chromophore Schiff base is deprotonated but the conformational change is small. ${ }^{16,} 33$ To examine whether the conformational change in the membrane agrees with that in the crystal, the scattering curves of the intermediates were calculated from the crystal structures using CRYSOL. ${ }^{30}$

The scattering change for formation of Meta-II was calculated, using Met1-Asn326 and chromophore retinal in a-chain of $1 \mathrm{U} 19$ and 3PXO. 2I37 is trimeric and the structures are not homogeneous. Therefore, the scattering curves of c-chains of 2I37 and 2I36 were calculated, as c-chains undergo the largest conformational change among the three protomers. Because several amino acid residues were missing in $2 \mathrm{I} 37$ and 2I36, scattering profiles were calculated using Met1-Gly121, Ser127-Val230, Ala235, and Thr242-Cys322.

It was deduced that the large intensity change occurs due to the structural difference between 3PXO and 1U19, where elongation of helix V and outward tilt of helix VI occur. This intensity difference curve agreed with the experimental curve of $\mathrm{Rh} / \mathrm{ND}$, while the correction for the lipid bilayer ${ }^{17,18}$ was not carried out. On the other hand, scattering change calculated from $2 \mathrm{I} 37$ and $2 \mathrm{I} 36$ showed a significantly reduced peak at $0.2 \mathrm{~A}^{-1}$. This curve was similar to those of $\mathrm{Rh} / \mathrm{DDM}$ and $2 \mathrm{Rh} / \mathrm{ND}$, suggesting that structural changes are suppressed in these samples. This is consistent with the previous finding that the Gt activation efficiency of Meta-II in $2 \mathrm{Rh} / \mathrm{ND}$ and DDM $(0.01 \%)$ is significantly lower than that of Rh/ND. ${ }^{28}$ It is suspected that rhodopsin forms a dimer in $2 \mathrm{Rh} / \mathrm{ND}$, and that this dimerization suppresses conformational change. Reduced intensity in $\mathrm{Rh} / \mathrm{DDM}$ also suggests the dimerization of rhodopsin in $0.02 \% \mathrm{DDM}^{36}$ However, the interhelical interference between the two protomers may reduce the scattering intensity change. To investigate this, we constructed the dimer models, and the intensity difference curves representing helical movement in the dimer 
were deduced by CRYSOL.

The typical dimer forms of rhodopsin (1U19 and 1N3M) and opsin (3CAP) reported so far are shown in Figure 6. While $1 \mathrm{U} 19$ is an antiparallel dimer in which one protomer is flipped, 3CAP (a- and b-chains) and 1N3M (a- and c-chains) are parallel dimers which are likely to be present in the native disk membrane. Because the scattering curve predicted from the structure of Meta-II (3PXO) was indistinguishable from that from opsin (3CAP), we used opsin structure for the active conformation. In 3CAP, the helices VIII of the two protomers face each other (face-to-face parallel dimer), but they face away from each other in $1 \mathrm{~N} 3 \mathrm{M}$ (back-to-back parallel dimer) (Figures 6a and 6b, respectively). Using these structures as the template, the antiparallel dimer of opsin, face-to-face parallel dimer of the dark state, and back-to-back parallel dimer of opsin were generated using PyMOL (The PyMOL Molecular Graphics System, Version 1.7.0 Schrödinger, LLC), as follows.

The a-chain of 1 U19 (Met1-Asn326) was superimposed on the a-chain of 3CAP (Met1Asn326) using the "align" command of PyMOL. Similarly, b-chain of 1 U19 was superimposed on the b-chain of 3CAP. Aligned a- and b-chains of 1 U19 were used to predict the scattering curve of the face-to-face parallel dimer of dark state (Figure 6a). Similarly, aand b-chains of 3CAP were superimposed on a- and c-chains of 1N3M (Met1-Asn326) to generate the back-to-back parallel dimer of opsin (Figure 6b), and a- and b-chains of 3CAP were superimposed on a- and b-chains of 1 U19 (Met1-Asn326), to generate the antiparallel dimer of opsin (Figure 6c).

The intensity difference curves for these conformational changes in the dimer were calculated using Met1-Asn326, and compared with the intensity difference curve of the monomer (Figure 6, bottom). In all dimers, it was found that the characteristic peak at $0.2 \AA^{-1}$ was not reduced, and the valley at $0.6 \AA^{-1}$ was enhanced unlike in the experimental curve, strongly suggesting that the reduced intensity difference in $2 \mathrm{Rh} / \mathrm{ND}$ was not derived from the interhelical interference between two protomers. It is likely that the structural change is suppressed in the dimer. However, since there appears to be enough space for outward movement of the helix VI (Figure 6), conformational change should not be suppressed simply by steric hindrance between the protomers.

These experiments show that the kinetics of the photobleaching process, the equilibrium between Meta-I and Meta-II, and the conformational change of rhodopsin in 2Rh/ND are different from those of $\mathrm{Rh} / \mathrm{ND}$. Therefore, it is likely that there is an interaction between two rhodopsin molecules tethered in ND, which suppresses the helical rearrangement upon photoactivation. In our $2 \mathrm{Rh} / \mathrm{ND}$ sample, however, the orientation of the rhodopsin molecule 
was not controlled, and therefore both the parallel and anti-parallel dimer would have been present. ${ }^{31}$ Physiologically, Meta-II is likely to be contained in the parallel Meta-II/rhodopsin heterodimer. Therefore, the relationship between the architecture of the dimer and the conformational change should be further investigated to elucidate the physiological relevance of the formation of the dimer.

\section{Acknowledgements}

Part of this research is based on the Cooperative Research Project of Research Institute of Electronics, Shizuoka University. The synchrotron radiation experiments were performed at the BL40B2 in the SPring-8, with the approval of the Japan Synchrotron Radiation Research Institute (JASRI) (Proposal No. 2011A1245, 2012A1263, 2012B1307, 2013A1136, 2014A1247, and 2014B1219). 


\section{REFERENCES}

1 K. Palczewski, T. Kumasaka, T. Hori, C. A. Behnke, H. Motoshima, B. A. Fox, I. Le Trong, D. C. Teller, T. Okada, R. E. Stenkamp, M. Yamamoto and M. Miyano, Crystal structure of rhodopsin: A G protein-coupled receptor, Science, 2000, 289, 739.

2 X. Deupi and J. Standfuss, Structural insights into agonist-induced activation of Gprotein-coupled receptors, Curr. Opin. Struct. Biol., 2011, 21, 541.

3 J. H. Park, P. Scheerer, K. P. Hofmann, H. W. Choe and O. P. Ernst, Crystal structure of the ligand-free G-protein-coupled receptor opsin, Nature, 2008, 454, 183.

4 P. Scheerer, J. H. Park, P. W. Hildebrand, Y. J. Kim, N. Krauss, H. W. Choe, K. P. Hofmann and O. P. Ernst, Crystal structure of opsin in its G-protein-interacting conformation, Nature, 2008, 455, 497.

5 H. W. Choe, Y. J. Kim, J. H. Park, T. Morizumi, E. F. Pai, N. Krauss, K. P. Hofmann, P. Scheerer and O. P. Ernst, Crystal structure of metarhodopsin II, Nature, 2011, 471, 651.

6 X. Deupi, P. Edwards, A. Singhal, B. Nickle, D. Oprian, G. Schertler and J. Standfuss, Stabilized G protein binding site in the structure of constitutively active metarhodopsin-II, Proc. Natl. Acad. Sci. U. S. A., 2012, 109, 119.

7 J. Standfuss, P. C. Edwards, A. D'Antona, M. Fransen, G. Xie, D. D. Oprian and G. F. Schertler, The structural basis of agonist-induced activation in constitutively active rhodopsin, Nature, 2011, 471, 656.

8 D. Salom, D. T. Lodowski, R. E. Stenkamp, I. Le Trong, M. Golczak, B. Jastrzebska, T. Harris, J. A. Ballesteros and K. Palczewski, Crystal structure of a photoactivated deprotonated intermediate of rhodopsin, Proc. Natl. Acad. Sci. U. S. A., 2006, 103, 16123.

9 S. G. Rasmussen, B. T. DeVree, Y. Zou, A. C. Kruse, K. Y. Chung, T. S. Kobilka, F. S. Thian, P. S. Chae, E. Pardon, D. Calinski, J. M. Mathiesen, S. T. Shah, J. A. Lyons, M. Caffrey, S. H. Gellman, J. Steyaert, G. Skiniotis, W. I. Weis, R. K. Sunahara and B. K. Kobilka, Crystal structure of the $\beta_{2}$ adrenergic receptor-Gs protein complex, Nature, 2011, 477, 549.

10 A. K. Shukla, A. Manglik, A. C. Kruse, K. Xiao, R. I. Reis, W. C. Tseng, D. P. Staus, D. Hilger, S. Uysal, L. Y. Huang, M. Paduch, P. Tripathi-Shukla, A. Koide, S. Koide, W. I. Weis, A. A. Kossiakoff, B. K. Kobilka and R. J. Lefkowitz, Structure of active $\beta$-arrestin-1 bound to a G-protein-coupled receptor phosphopeptide, Nature, 2013, 497, 137.

11 R. Maeda, M. Hiroshima, T. Yamashita, A. Wada, S. Nishimura, Y. Sako, Y. Shichida and Y. Imamoto, Single-molecule observation of the ligand-induced population shift of rhodopsin, a G-protein-coupled receptor, Biophys. J., 2014, 106, 915.

12 R. Vogel and F. Siebert, Conformations of the active and inactive states of opsin, $J$. Biol. Chem., 2001, 276, 38487.

13 B. Knierim, K. P. Hofmann, O. P. Ernst and W. L. Hubbell, Sequence of late molecular events in the activation of rhodopsin, Proc. Natl. Acad. Sci. U. S. A., 2007, 104, 20290.

14 M. Mahalingam, K. Martinez-Mayorga, M. F. Brown and R. Vogel, Two protonation switches control rhodopsin activation in membranes, Proc. Natl. Acad. Sci. U. S. A., 2008, 105, 17795.

15 Y. Yamazaki, T. Nagata, A. Terakita, H. Kandori, Y. Shichida and Y. Imamoto, Mapping of the local environmental changes in proteins by cysteine scanning, 
Biophysics, 2014, 10, 1.

16 Y. Yamazaki, T. Nagata, A. Terakita, H. Kandori, Y. Shichida and Y. Imamoto, Intramolecular interactions that Induce helical rearrangement upon rhodopsin activation: Light-induced structural changes in metarhodopsin $\mathrm{II}_{\mathrm{a}}$ probed by cysteine S-H stretching vibrations, J. Biol. Chem., 2014, 289, 13792.

17 E. Malmerberg, M. B.-G. PH, G. Katona, X. Deupi, D. Arnlund, C. Wickstrand, L. C. Johansson, S. Westenhoff, E. Nazarenko, X. S. GF, A. Menzel, W. J. de Grip and R. Neutze, Conformational activation of visual rhodopsin in native disc membranes, Science signaling, 2015, 8, ra26.

18 M. Andersson, E. Malmerberg, S. Westenhoff, G. Katona, M. Cammarata, A. B. Wohri, L. C. Johansson, F. Ewald, M. Eklund, M. Wulff, J. Davidsson and R. Neutze, Structural dynamics of light-driven proton pumps, Structure, 2009, 17, 1265.

19 M. Kataoka and Y. Goto, X-ray solution scattering studies of protein folding, Fold. Des., 1996, 1, R107.

20 T. H. Bayburt, Y. V. Grinkova and S. G. Sligar, Self-assembly of discoidal phospholipid bilayer nanoparticles with membrane scaffold proteins, Nano Lett., 2002, 2, 853.

21 K. Kojima, Y. Imamoto, R. Maeda, T. Yamashita and Y. Shichida, Rod visual pigment optimizes active state to achieve efficient $\mathrm{G}$ protein activation as compared with cone visual pigments, J. Biol. Chem., 2014, 289, 5061.

22 H. Tsukamoto, A. Sinha, M. DeWitt and D. L. Farrens, Monomeric rhodopsin is the minimal functional unit required for arrestin binding, J. Mol. Biol., 2010, 399, 501.

23 Y. Imamoto, I. Seki, T. Yamashita and Y. Shichida, Efficiencies of activation of transducin by cone and rod visual pigments, Biochemistry, 2013, 52, 3010-3018.

24 Y. Imamoto and Y. Shichida, Thermal recovery of iodopsin from photobleaching intermediates, Photochem. Photobiol., 2008, 84, 941.

25 F. J. Bartl and R. Vogel, Structural and functional properties of metarhodopsin III: recent spectroscopic studies on deactivation pathways of rhodopsin, Phys. Chem. Chem. Phys., 2007, 9, 1648.

26 B. König, W. Welte and K. P. Hofmann, Photoactivation of rhodopsin and interaction with transducin in detergent micelles. Effect of 'doping' with steroid molecules, FEBS Lett., 1989, 257, 163.

27 J. H. Parkes and P. A. Liebman, Temperature and $\mathrm{pH}$ dependence of the metarhodopsin I-metarhodopsin II kinetics and equilibria in bovine rod disk membrane suspensions, Biochemistry, 1984, 23, 5054.

28 T. H. Bayburt, A. J. Leitz, G. Xie, D. D. Oprian and S. G. Sligar, Transducin activation by nanoscale lipid bilayers containing one and two rhodopsins, J. Biol. Chem., 2007, 282, 14875.

29 T. Kawaguchi, T. Hamanaka and Y. Kito, X-ray diffraction pattern from internal structure of bovine rhodopsin, J. Biochem., 1996, 119, 396.

30 D. I. Svergun, C. Baberato and M. H. J. Koch, CRYSOL-a program to evaluate X-ray solution scattering of biological macromolecules from atomic coordinates, J. Appl. Crystallogr., 1995, 28, 768.

31 S. Banerjee, T. Huber and T. P. Sakmar, Rapid incorporation of functional rhodopsin into nanoscale apolipoprotein bound bilayer (NABB) particles, J. Mol. Biol., 2008, 377, 1067.

32 J. J. Ruprecht, T. Mielke, R. Vogel, C. Villa and G. F. X. Schertler, Electron crystallography reveals the structure of metarhodopsin I, EMBO J., 2004, 23, 3609.

33 E. Zaitseva, M. F. Brown and R. Vogel, Sequential rearrangement of interhelical networks upon rhodopsin activation in membranes: the Meta IIa conformational 
substate, J. Am. Chem. Soc., 2010, 132, 4815.

34 H. Nakamichi and T. Okada, Crystallographic analysis of primary visual photochemistry, Angew. Chem. Int. Ed. Engl., 2006, 45, 4270.

35 H. Nakamichi and T. Okada, Local peptide movement in the photoreaction intermediate of rhodopsin, Proc. Natl. Acad. Sci. U. S. A., 2006, 103, 12729.

36 B. Jastrzebska, T. Maeda, L. Zhu, D. Fotiadis, S. Filipek, A. Engel, R. E. Stenkamp and K. Palczewski, Functional characterization of rhodopsin monomers and dimers in detergents, J. Biol. Chem., 2004, 279, 54663. 
Table 1: Spectroscopic characterization of rhodopsin samples

\begin{tabular}{cccccc}
\hline \multirow{2}{*}{ Sample } & \multicolumn{2}{c}{ Meta-II formation } & & Meta-III formation & Meta-I:Meta-II \\
\cline { 2 - 3 } & $\tau_{1}(\mathrm{~s})$ & $\tau_{2}(\mathrm{~s})$ & & $\tau_{3}(\mathrm{~s})$ & $13: 87$ \\
\hline $\mathrm{Rh} / \mathrm{DDM}$ & 0.0004 & 0.0061 & & 1237 & $20: 80$ \\
$\mathrm{Rh} / \mathrm{ND}$ & 0.0060 & 0.0664 & & 923 & $30: 70$ \\
2Rh/ND & 0.0160 & 0.1676 & & 945 & \\
\hline
\end{tabular}


Figure Legends

Figure 1: Photobleaching process of rhodopsin, in DDM and ND. The transient difference spectra after photoactivation were recorded over a $10^{-4}$ to $10^{3}-\mathrm{sec}$ time scale. Decayassociated difference spectra (DADS) for Rh/DDM (a), Rh/ND (b), and 2Rh/ND (c) were obtained by SVD analysis. The time constants for DADS1 (red), DADS2 (green), and DADS3 (blue) are shown in Table 1. Extrapolated difference spectra for $t=\infty$ (DADS0) are also presented (black).

Figure 2: The composition of Meta-I and Meta-II in the equilibrium. The difference spectra between Meta-I/Meta-II equilibrium mixture and dark state were calculated by subtracting DADS3 from DADS0 $(t=\infty)$ in Figure 1 (red). They were decomposed into the model difference spectra between Meta-I and rhodopsin (green), and between Meta-II and rhodopsin (blue). The resulting spectra (sum of model spectra, black) were in good agreement with the experimental ones (red). The ratios of Meta-I and Meta-II are shown in Table 1.

Figure 3: X-ray scattering profile in the high-angle region. The scattering curves of $\mathrm{Rh} / \mathrm{DDM}$, $\mathrm{Rh} / \mathrm{ND}$, and $2 \mathrm{Rh} / \mathrm{ND}$ were measured before (red) and after (blue) the photoactivation of rhodopsin. The scattering curves are presented using a logarithmic scale, and offset for comparison. The scattering curves of the rhodopsin monomer (a-chain of 1U19) and dimer (a- and b-chains of 1U19) were calculated by CRYSOL (green).

Figure 4: X-ray radiation damage on ND. $(a, b)$ Intensity difference curves before and after photoactivation of $\mathrm{Rh} / \mathrm{ND}$ (a) and empty ND (b) were calculated using the averaged curves of four HAXS, curves before and after photoactivation. Intensity difference curves for three typical datasets are presented. (c) To obtain the intensity difference $(\Delta I)$ just before and after photoactivation, the intensities at given $Q$ before $(n=1,2,3,4)$ and after photoactivation $(n=$ $5,6,7,8)$ were plotted, and extrapolated to $n=4.5$. The example for $Q=0.22 \AA^{-1}$ is presented. Populations of rhodopsin, Meta-I, Meta-II, and Meta-III estimated by the UVvisible spectroscopy are illustrated below. For more details, see text. (d, e) $\Delta I$ for Rh/ND (d) and empty ND (e), obtained as shown in (c), are plotted against $Q$. Intensity difference curves for three typical datasets are presented. 
Figure 5: Light-induced X-ray scattering changes of rhodopsin. The intensity was normalized by the amount of Meta-II, assuming that the scattering changes due to the formation of MetaI and Meta-III were substantially smaller than those due to the formation of Meta-II. The intensity difference curves were calculated using the crystal structures of Meta-II (3PXO) and rhodopsin (1U19), or those of deprotonated intermediate (Meta-II') (2I37, c-chain) and rhodopsin (2I36, c-chain).

Figure 6: Model of the conformational change in the rhodopsin dimer. (a) Dark state structure (1U19, red) was superimposed on the structure if the face-to-face parallel dimer of opsin (3CAP, yellow). (b) Opsin structure (3CAP, yellow) was superimposed on the structure of the back-to-back parallel dimer of rhodopsin (a- and c-chains of 1N3M, red). (c) Opsin structure (3CAP, yellow) was superimposed on the structure of the antiparallel dimer of rhodopsin (1U19, red). The intensity difference curves predicted for the photoactivation of the rhodopsin dimers (red), in comparison with that of the rhodopsin monomer (blue), are shown below. 
Figure 1

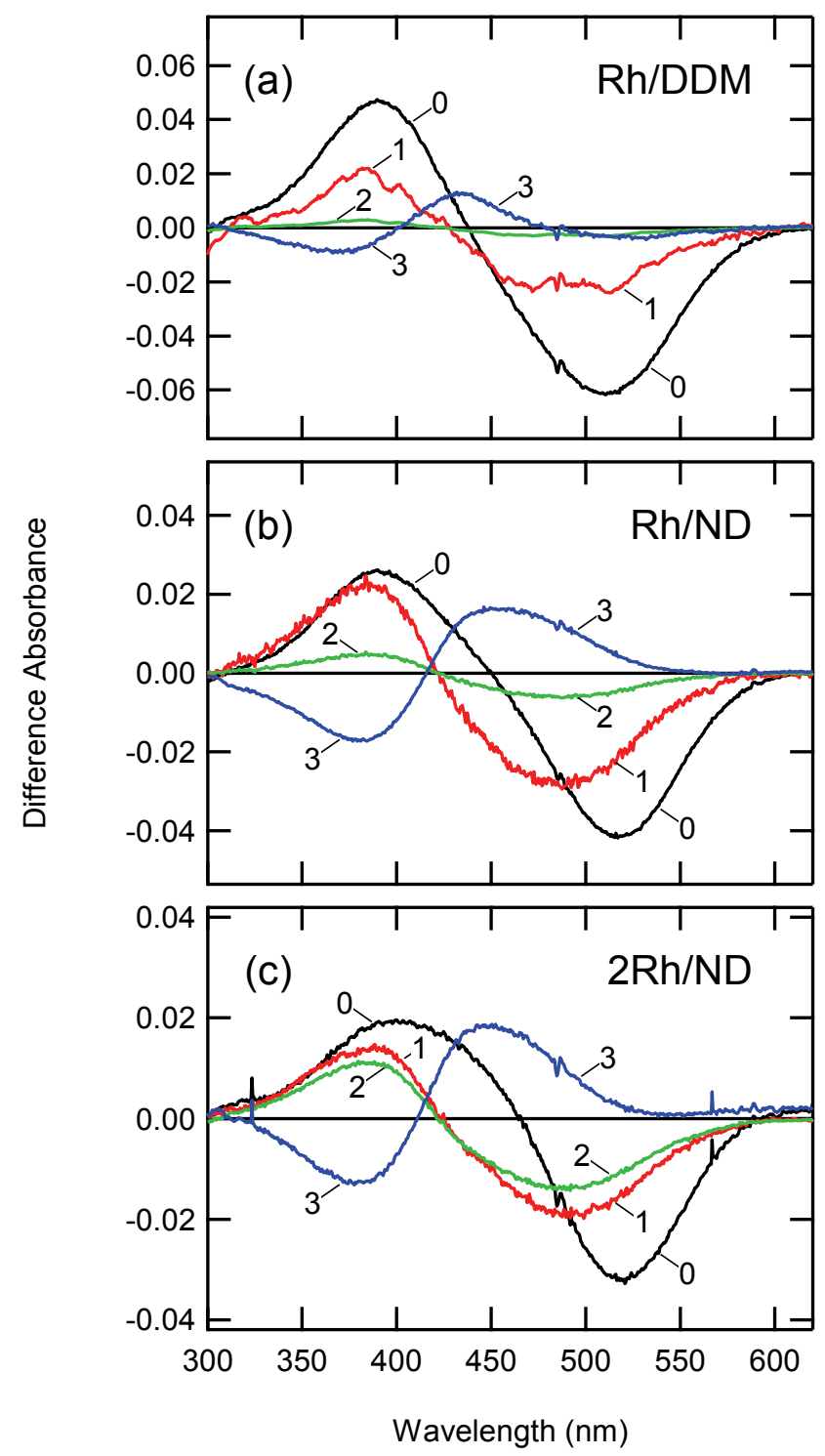


Figure 2

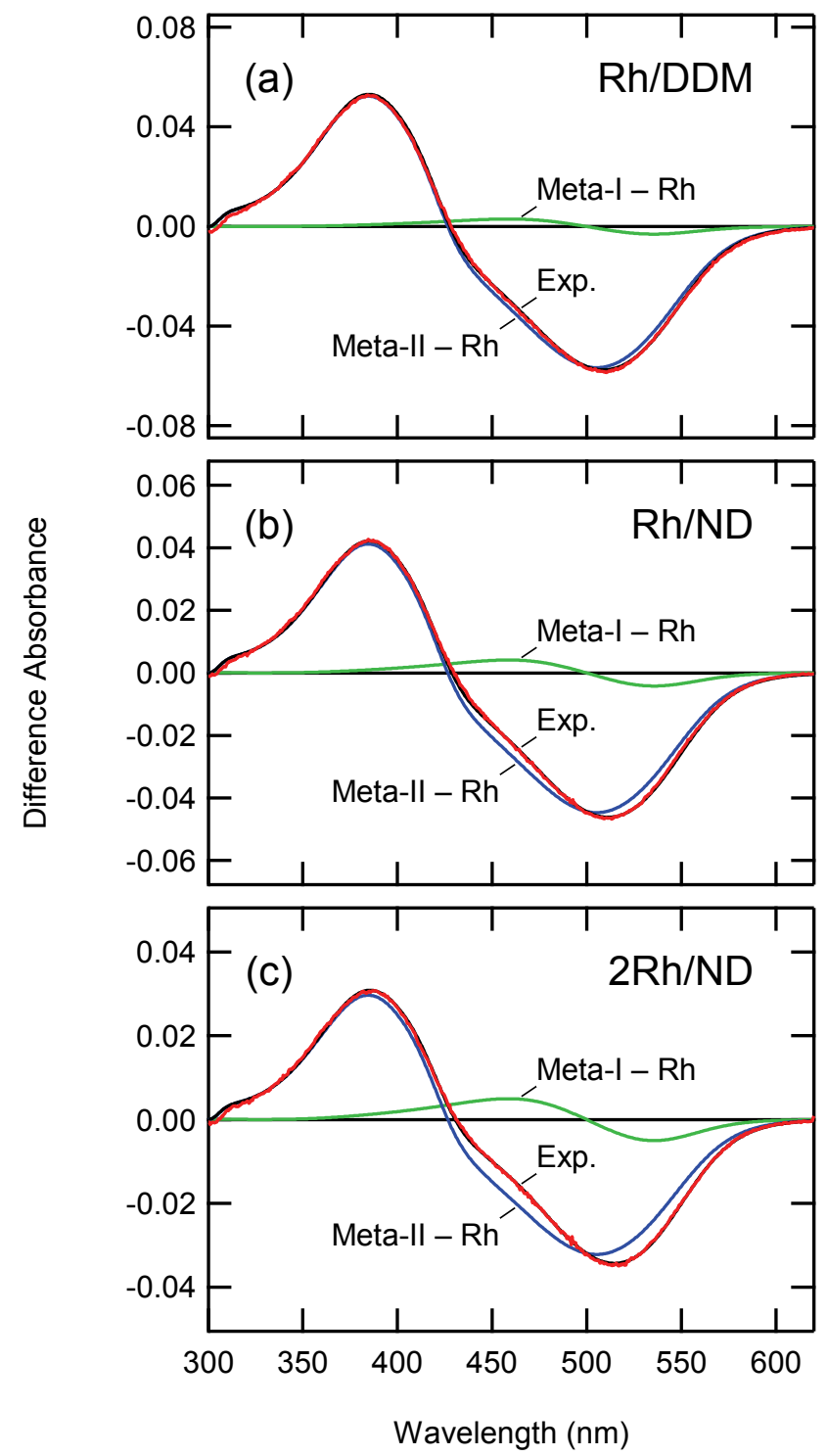


Figure 3

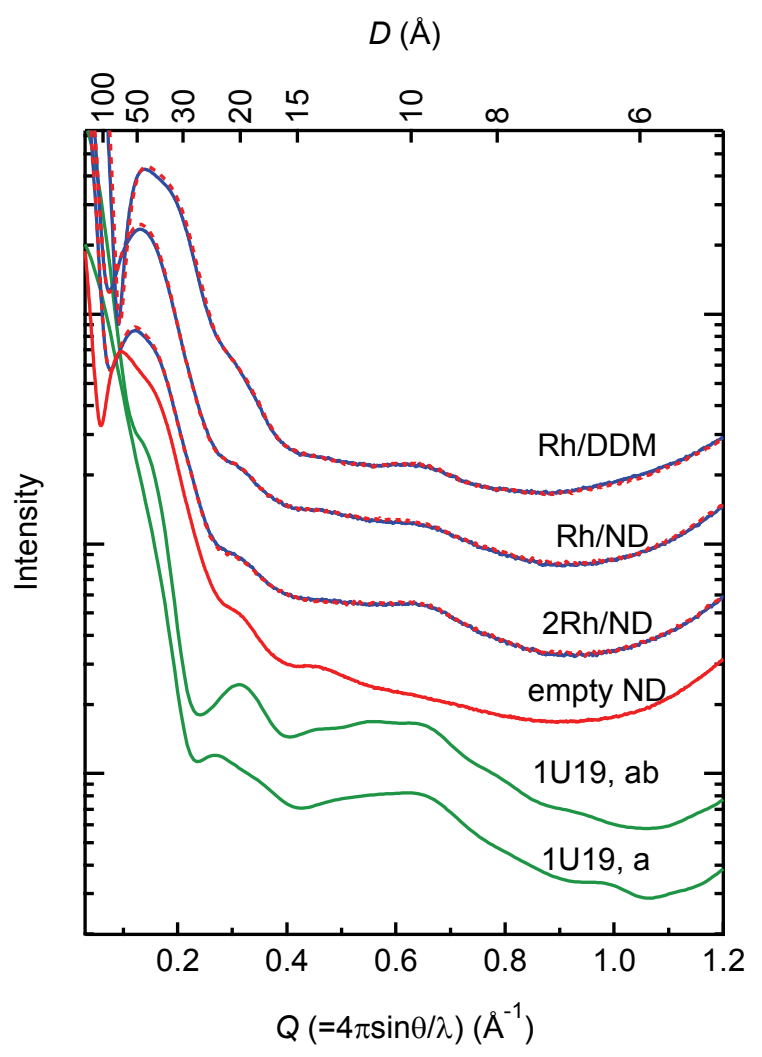


Figure 4
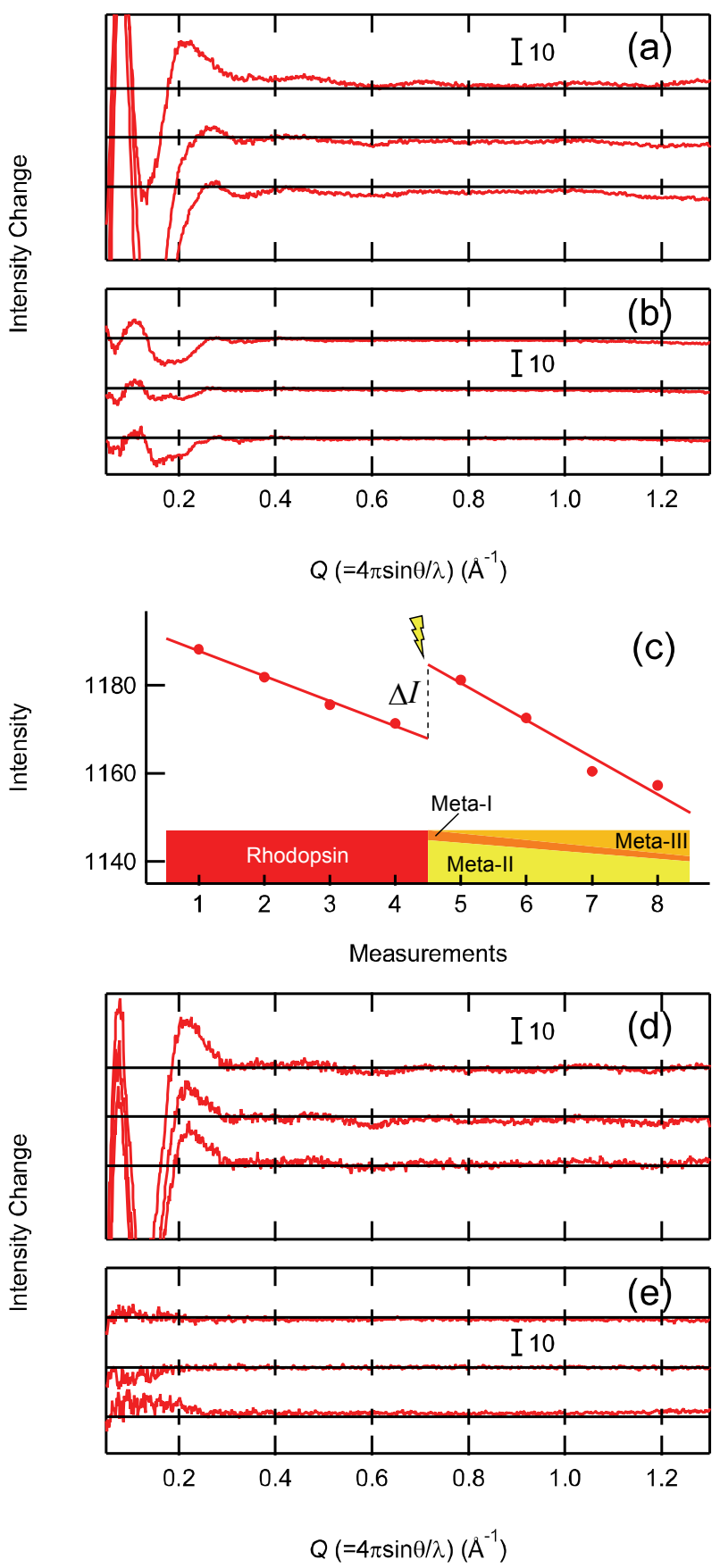
Figure 5

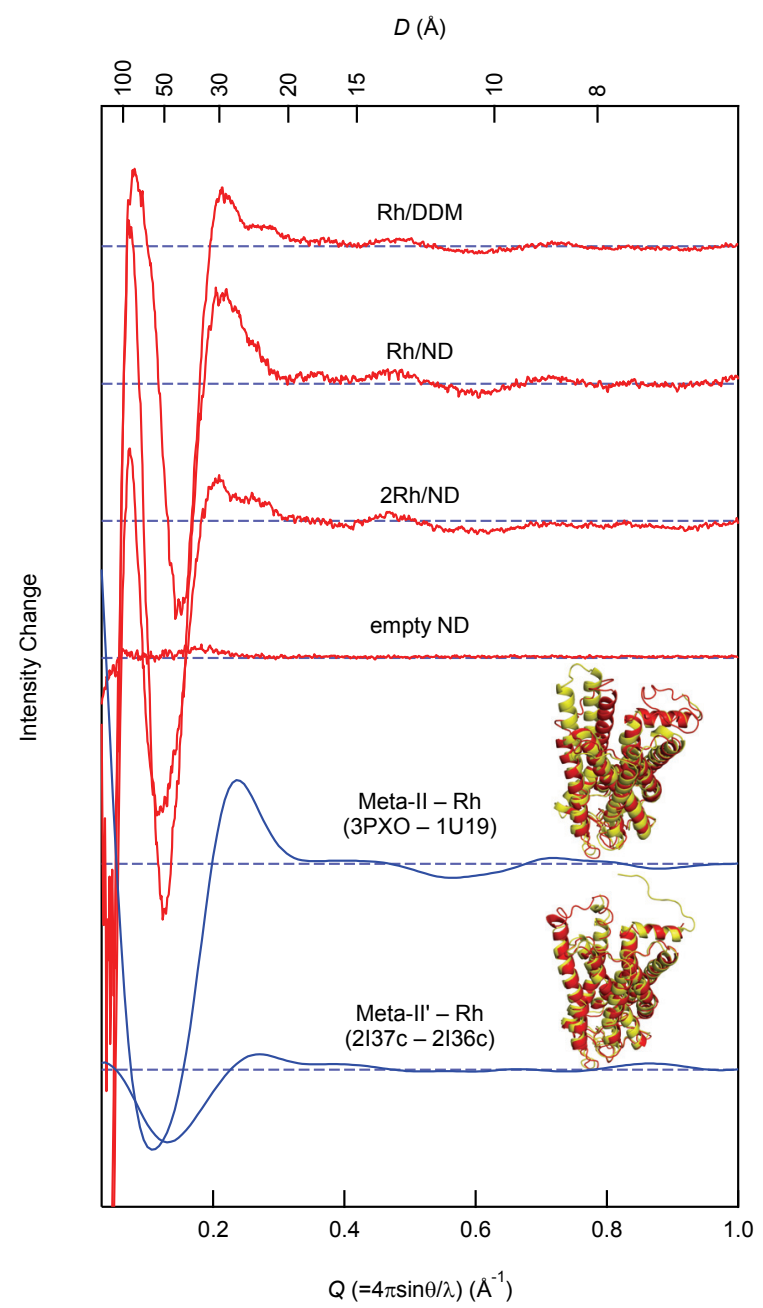


Figure 6

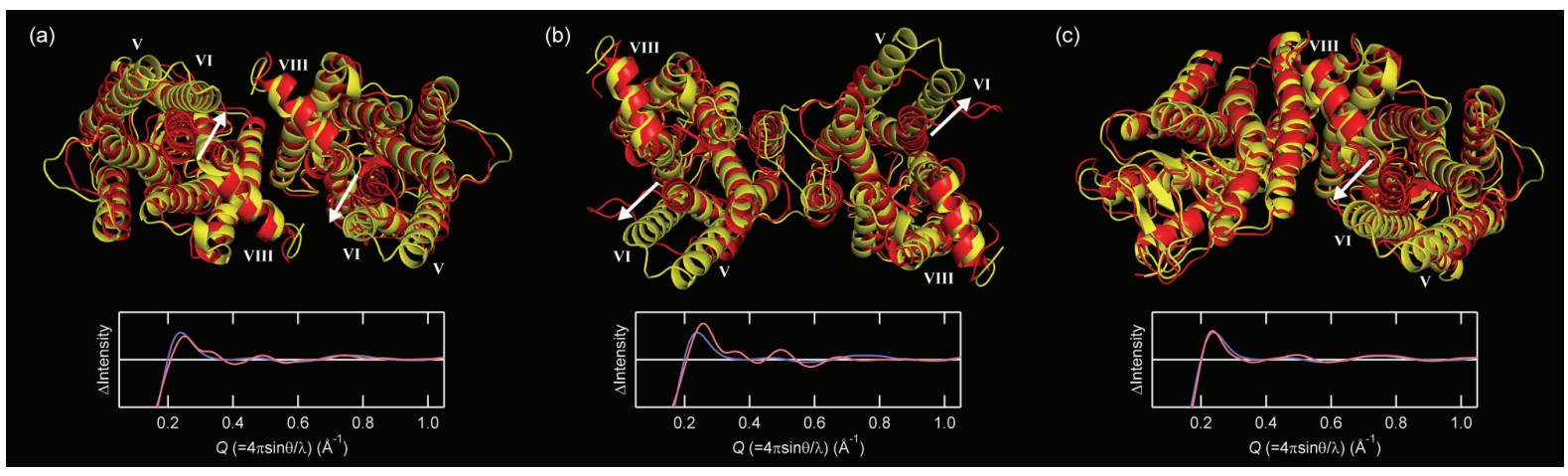




\section{Graphic Abstract}

Light-induced conformational change of monomeric and dimeric rhodopsin in the nanodisc membrane was directly monitored by high-angle solution X-ray scattering.

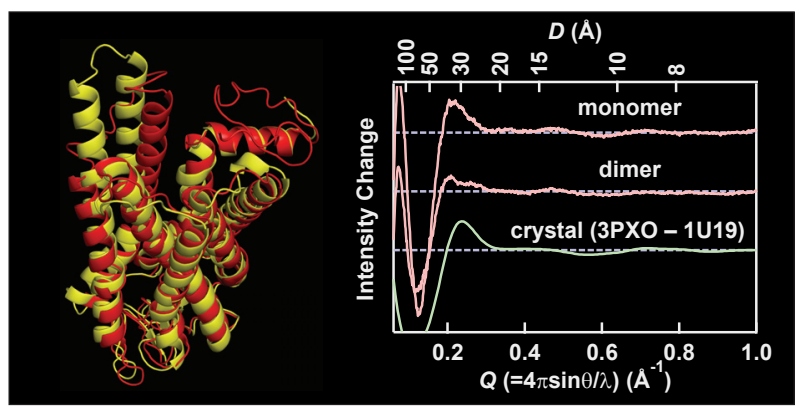

